\title{
Editorial
}

\section{PARPs, PAR and NAD Metabolism and Their Inhibitors in Cancer}

\author{
Nicola Curtin ${ }^{1, *(D)}$ and Péter Bai ${ }^{2,3,4, * \text { (D) }}$ \\ 1 Newcastle University Centre for Cancer, Translational and Clinical Research Institute, \\ Faculty of Medical Sciences, Newcastle University, Newcastle upon Tyne NE2 4HH, UK \\ 2 Department of Medical Chemistry, Faculty of Medicine, University of Debrecen, 4032 Debrecen, Hungary \\ 3 MTA-DE Lendület Laboratory of Cellular Metabolism, 4032 Debrecen, Hungary \\ 4 Research Center for Molecular Medicine, Faculty of Medicine, University of Debrecen, \\ 4032 Debrecen, Hungary \\ * Correspondence: nicola.curtin@newcastle.ac.uk (N.C.); baip@med.unideb.hu (P.B.); \\ Tel.: +36-52-412-345 (P.B.); Fax: +36-52-412-566 (P.B.)
}

Received: 15 September 2020; Accepted: 19 October 2020; Published: 24 November 2020

check for updates

The role of poly(ADP-ribose) polymerase-1 (PARP1) in DNA repair and as a potential target for anticancer therapy has been under investigation for more than 50 years. The field has expanded over the decades to include not only a family of ADP-ribosylating enzymes (PARPs/ARTDs), but also interacting and polymer-degrading proteins. In this special issue of Cancers primary research articles and reviews describe various aspects of PARP biology along with therapeutic targeting. Some historical perspective is reviewed along with the development of the PARP inhibitor (PARPi), rucaparib/Rubraca ${ }^{\circledR}$, including the identification of the synthetic lethality with homologous recombination repair (e.g., BRCA) defects [1]. Further consideration of the mode of action of several PARPi and their interaction with cytotoxic chemotherapy is described by Min and Im [2], and the evidence for a unique mode-of-action of another PARPi, PJ34 [3] is summarized. Several other aspects of PARPi therapy are described, including research indicating the therapeutic potential of the PARPi, olaparib, as a single agent in myelodysplastic syndromes, not only through cytotoxic and cytostatic effects but also through the induction of differentiation [4], along with the augmentation of UVB-induced DNA damage and mitochondrial alterations resulting in reduced proliferation and viability of keratinocytes [5]. On the other side of the coin, deficiency of the enzyme that degrades the PARP-generated ADP-ribose polymers, PARG, in ES cells resulted delayed tumour growth when they were implanted SC and increased antitumour activity of X-rays [6].

Moving PARPi therapy beyond BRCA mutated cancers requires the use of biomarkers to predict PARPi sensitivity as reviewed by Singh et al. [7]. The role of defects in the G1 cell cycle checkpoint signaling kinase, ATM, as a determinant of sensitivity to PARPi was reviewed, with the finding that PARPi alone are cytostatic in ATM defective cancer cells but require the addition of an inhibitor of the S/G2 cell cycle checkpoint kinase, ATR, to induce cell death [8]. An investigation of the synergy between the PARPi, olaparib, and the ATR inhibitor VE-821 in a panel of neuroblastoma cells, revealed that it was independent of MYCN or ATM status in these cells [9] and similar studies identified that the synergy between an inhibitor of the S/G2 cell cycle checkpoint kinase, CHK1, and the PARPi, rucaparib was largely through the impairment of homologous recombination repair by the CHK1 inhibitor [10]. Depletion of p60/150 CAF-1 also impaired homologous recombination repair thereby inducing sensitivity to PARPi and irradiation in head and neck cancers with therapeutic implications [11]. Interestingly, depletion of NMNAT1, which is involved in the synthesis of NAD+, PARP's substrate, induced DNA damage and sensitized cells to cisplatin but exhibited redundancy with PARPi in this respect [12]. Inhibition of EGFR or Syk (which mediates EGFR signaling) was 
synergistically cytotoxic in combination with olaparib in squamous cell carcinoma cells suggesting therapeutic potential [13].

Of course, resistance to PARPi is an emerging problem clinically and the role of the PI3K-AKT pathway in protection from PARPi-induced cytotoxicity and its significance in shock, inflammation, ischemia-reperfusion injury and cancer is reviewed by Gallyas et al. [14]. Interestingly, gastric cancer cell lines made resistant to olaparib were found to be cross-resistant to cisplatin, but had increased sensitivity to irinotecan due to upregulation of TOP1 and TDP1, which has therapeutic implications for patients who develop PARPi resistance [15].

PARP has been investigated as a regulator of transcription and key roles regarding the role of PARP both in nucleolar function in relation to cancer biology and the role PARylation plays in the regulation of transcription were reviewed $[16,17]$. With original research showing that PARylation activates the histone acetyl transferase, EP300, contributing to its regulation of transcription of DNA repair and proliferation genes [18].

An important factor in cancer biology is the tumour microenvironment and the interaction between PARP and key features of the tumour microenvironment such as autophagy, hypoxia and angiogenesis was reviewed [19]. There is significant interest in the immune microenvironment and the roles of both PARP1 and PARP2 in modulating both the innate and adaptive immune system was reviewed by Yelamos et al. [20], with the therapeutic potential of the combination of PARPi with immune checkpoint inhibitors, including translational and clinical studies were reviewed by Peyraud and Italiano [21].

Feijs and colleagues [22] reviewed the roles of ADP-ribosyl hydrolases, also involved in the degradation of ADP-ribose and poly(ADP-ribose) chains, MACROD1, MACROD2 and TARG1 in carcinogenesis. The role of ARH1, another degradatory enzyme, was reviewed by Ishiwata-Endo and colleagues [23] in cancer and non-cancer diseases.

The roles for poly(ADP-ribosyl)ation in biochemistry, cell biology, physiology and pathophysiology are rapidly expanding, the findings discussed in the "PARPs, PAR and NAD Metabolism and Their Inhibitors in Cancer" special issue of Cancers will surely provide a better understanding of these processes and widen the scope of our appreciation of poly(ADP-ribosyl)ation.

Funding: P.B. was supported by grants from NKFIH (K123975 and GINOP-2.3.2-15-2016-00006) and by the Higher Education Institutional Excellence Programme (NKFIH-1150-6/2019) of the Ministry of Innovation and Technology in Hungary, within the framework of the Biotechnology thematic programme of the University of Debrecen. NC is funded by Newcastle University.

Conflicts of Interest: N.C. has worked for many years on the development of rucaparib resulting in 2 patents WO 2005/012305 A2 andWO/2006/033006 with royalties paid to CRUK and Newcastle University through which she receives a percentage. She has received research funding foe PARP-related studies from Agouron Pharmaceuticals, Pfizer, BioMarin and consulted for Abbvie, Eisai and Tesaro.

\section{References}

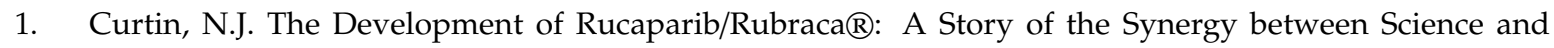
Serendipity. Cancers 2020, 12, 564. [CrossRef] [PubMed]

2. Min, A.; Im, S.A. PARP Inhibitors as Therapeutics: Beyond Modulation of PARylation. Cancers 2020, $12,394$. [CrossRef] [PubMed]

3. Cohen-Armon, M. The Modified Phenanthridine PJ34 Unveils an Exclusive Cell-Death Mechanism in Human Cancer Cells. Cancers 2020, 12, 1628. [CrossRef] [PubMed]

4. Faraoni, I.; Consalvo, M.I.; Aloisio, F.; Fabiani, E.; Giansanti, M.; Di Cristino, F.; Falconi, G.; Tentori, L.; Di Veroli, A.; Curzi, P.; et al. Cytotoxicity and Differentiating Effect of the Poly(ADP-Ribose) Polymerase Inhibitor Olaparib in Myelodysplastic Syndromes. Cancers 2019, 11, 1373. [CrossRef] [PubMed]

5. Hegedûs, C.; Boros, G.; Fidrus, E.; Kis, G.N.; Antal, M.; Juhász, T.; Janka, E.A.; Jankó, L.; Paragh, G.; Emri, G.; et al. PARP1 Inhibition Augments UVB-Mediated Mitochondrial Changes-Implications for UV-Induced DNA Repair and Photocarcinogenesis. Cancers 2019, 12, 5. [CrossRef] [PubMed] 
6. Sonoda, Y.; Sasaki, Y.; Gunji, A.; Shirai, H.; Araki, T.; Imamichi, S.; Onodera, T.; Rydén, A.M.; Watanabe, M.; Itami, J.; et al. Reduced Tumorigenicity of Mouse ES Cells and the Augmented Anti-Tumor Therapeutic Effects under Parg Deficiency. Cancers 2020, 12, 1056. [CrossRef]

7. Singh, N.; Pay, S.L.; Bhandare, S.B.; Arimpur, U.; Motea, E.A. Therapeutic Strategies and Biomarkers to Modulate PARP Activity for Targeted Cancer Therapy. Cancers 2020, 12, 972. [CrossRef]

8. Jette, N.R.; Kumar, M.; Radhamani, S.; Arthur, G.; Goutam, S.; Yip, S.; Kolinsky, M.; Williams, G.J.; Bose, P.; Lees-Miller, S.P. ATM-Deficient Cancers Provide New Opportunities for Precision Oncology. Cancers 2020, 12, 687. [CrossRef]

9. Southgate, H.E.D.; Chen, L.; Tweddle, D.A.; Curtin, N.J. ATR Inhibition Potentiates PARP Inhibitor Cytotoxicity in High Risk Neuroblastoma Cell Lines by Multiple Mechanisms. Cancers 2020, 12, 1095. [CrossRef]

10. Smith, H.L.; Prendergast, L.; Curtin, N.J. Exploring the Synergy between PARP and CHK1 Inhibition in Matched BRCA2 Mutant and Corrected Cells. Cancers 2020, 12, 878. [CrossRef]

11. Morra, F.; Merolla, F.; Picardi, I.; Russo, D.; Ilardi, G.; Varricchio, S.; Liotti, F.; Pacelli, R.; Palazzo, L.; Mascolo, M.; et al. CAF-1 Subunits Levels Suggest Combined Treatments with PARP-Inhibitors and Ionizing Radiation in Advanced HNSCC. Cancers 2019, 11, 1582. [CrossRef] [PubMed]

12. Kiss, A.; Ráduly, A.P.; Regdon, Z.; Polgár, Z.; Tarapcsák, S.; Sturniolo, I.; El-Hamoly, T.; Virág, L.; Hegedús, C. Targeting Nuclear NAD(+) Synthesis Inhibits DNA Repair, Impairs Metabolic Adaptation and Increases Chemosensitivity of U-2OS Osteosarcoma Cells. Cancers 2020, 12, 1180. [CrossRef] [PubMed]

13. Huang, D.Y.; Chen, W.Y.; Chen, C.L.; Wu, N.L.; Lin, W.W. Synergistic Anti-Tumour Effect of Syk Inhibitor and Olaparib in Squamous Cell Carcinoma: Roles of Syk in EGFR Signalling and PARP1 Activation. Cancers 2020, 12, 489. [CrossRef] [PubMed]

14. Gallyas, F., Jr.; Sumegi, B.; Szabo, C. Role of Akt Activation in PARP Inhibitor Resistance in Cancer. Cancers 2020, 12, 532. [CrossRef]

15. Kim, J.W.; Min, A.; Im, S.A.; Jang, H.; Kim, Y.J.; Kim, H.J.; Lee, K.H.; Kim, T.Y.; Lee, K.W.; Oh, D.Y.; et al. TDP1 and TOP1 Modulation in Olaparib-Resistant Cancer Determines the Efficacy of Subsequent Chemotherapy. Cancers 2020, 12, 334. [CrossRef]

16. Engbrecht, M.; Mangerich, A. The Nucleolus and PARP1 in Cancer Biology. Cancers 2020, 12, 1813. [CrossRef]

17. Páhi, Z.G.; Borsos, B.N.; Pantazi, V.; Ujfaludi, Z.; Pankotai, T. PARylation during Transcription: Insights into the Fine-Tuning Mechanism and Regulation. Cancers 2020, 12, 183. [CrossRef]

18. Sobczak, M.; Pitt, A.R.; Spickett, C.M.; Robaszkiewicz, A. PARP1 Co-Regulates EP300-BRG1-Dependent Transcription of Genes Involved in Breast Cancer Cell Proliferation and DNA Repair. Cancers 2019, 11, 1539. [CrossRef]

19. Martí, J.M.; Fernández-Cortés, M.; Serrano-Sáenz, S.; Zamudio-Martinez, E.; Delgado-Bellido, D.; Garcia-Diaz, A.; Oliver, F.J. The Multifactorial Role of PARP-1 in Tumor Microenvironment. Cancers 2020, 12, 739. [CrossRef]

20. Yélamos, J.; Moreno-Lama, L.; Jimeno, J.; Ali, S.O. Immunomodulatory Roles of PARP-1 and PARP-2: Impact on PARP-Centered Cancer Therapies. Cancers 2020, 12, 392. [CrossRef]

21. Peyraud, F.; Italiano, A. Combined PARP Inhibition and Immune Checkpoint Therapy in Solid Tumors. Cancers 2020, 12, 1502. [CrossRef] [PubMed]

22. Feijs, K.L.H.; Cooper, C.D.O.; Žaja, R. The Controversial Roles of ADP-Ribosyl Hydrolases MACROD1, MACROD2 and TARG1 in Carcinogenesis. Cancers 2020, 12, 604. [CrossRef] [PubMed]

23. Ishiwata-Endo, H.; Kato, J.; Stevens, L.A.; Moss, J. ARH1 in Health and Disease. Cancers 2020, $12,479$. [CrossRef] [PubMed]

Publisher's Note: MDPI stays neutral with regard to jurisdictional claims in published maps and institutional affiliations. 